\title{
2 Electrochemistry as a Powerful Tool for Creating Microstructures and Drawing Their Novel Functions
}

\author{
Yukio H. Ogata
}

Electrochemical methods are sometimes compared with other chemical, physical, and mechanical techniques to fabricate structures and to modify surfaces. Chemical processes utilize chemical reaction, where the rate is mainly controlled by the concentration of reactants and temperature, while physical or mechanical processes control the rate by physical parameters such as contact pressure, vapor pressure, kinetic energy, and temperature. Electrochemistry has a distinctive characteristic which thermal chemistry does not possess. The reaction and its rate can be controlled by electrical potential: the driving force or the free energy of reaction can be controlled electrically. The simple facility and the high processing rate are advantageous over the physical methods. These features greatly expand the capability of electrochemistry.

Electrochemistry requires electrodes to drive the reactions, where heterogeneous reaction proceeds. As a result, the reaction space is limited to 2-D instead of the 3-D space which is usual for homogeneous reactions. However, the 2-D feature becomes advantageous for structuring of electrode surfaces. An electrode itself can be a substrate to create an aimed structure by means of electrochemical deposition or dissolution reactions. Moreover, an environmental change induced by electrochemistry such as $\mathrm{pH}$ control can be another tool for the surface modification. These kinds of techniques, which have been utilizing in surface treatment of macro bodies for a long time, are basically applicable to the formation of microstructures down to nanometer-sizes. Novel functionality is often created by well-ordered structure and/or miniature size itself. Photonic-crystal structure is a typical example. The characteristic optical property is produced not by the material but solely by the structure. Luminescence in the visible region is observed on porous silicon at ambient temperature. The phenomenon is caused by the quantum confinement in nano-sized silicon structure. Enhancement of catalytic activity is also often reported in nano-sized surface. Some of the other examples will be found in this special issue.

Structuring requires nonuniformity on an electrode, and current distribution causes the nonuniformity. Local current density is induced by potential distribution and/or concentration distribution of related species, resulting in a change in the local reaction rate of dissolution, deposition, or surface modification. The resultant irregularity is sometimes unfavorable in electrochemical processes; however, electrochemical structure formation is based on such phenomenon. Deliberate disturbance in the distribution is utilized for microstructure formation, e.g. the use of a scanning microprobe with AFM, STM, or other modes is a powerful method. Defects induced in material also cause current distribution, and this phenomenon is utilized for patterning and structure formation. Masking is another important way to form structures. Electrochemical reactions occur only on a conductive surface. Masking with insulating materials restricts reaction sites to the uncovered part. Various lithographic techniques are applied to the micro-patterning. Natural lithography utilizing self-assembly attracts attention in nanotechnology, where the use of colloidal crystals and self-assembled monolayers is studied extensively. How to create well-controlled nonuniformity is an important issue for microstructure formation. Beautifully-controlled morphology, which is hardly created with artificial means, is easily attained in living beings. Utilization of the patterns as used in biomineralization is promising.

Basic techniques to make structures are deposition and dissolution, and they have been established in electrochemistry. The techniques and results accumulated by many researchers are applicable to the microstructure formation. We have to learn them earnestly. Meanwhile, the downsizing to microstructure requires much more precise and finer control of local current distribution. In addition, mass transfer, which determines local current density, in microstructures changes its picture, for example, mass transfer in a fine porous body often shows unexpected behavior. The deeper study and understanding are needed for the further development of electrochemical methods in nano- and micro-structure formation.

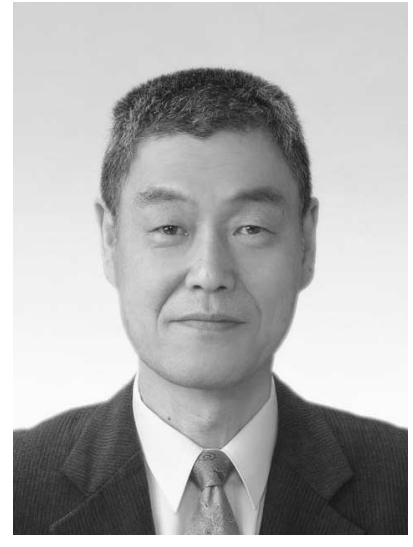

Yukio H. OGata

Professor

Institute of Advanced

Energy, Kyoto University 